\title{
Flood management in the low lands: from probability to risk
}

\author{
B. Broekhans \& A. F. Correljé \\ Delft University of Technology, \\ Faculty of Technology, Policy and Management, The Netherlands
}

\begin{abstract}
In deltas, populations always face flooding risks. Confronted with the high levels of rivers in the nineties, policy makers and scientists became involved in critical debates about the required level of security and possible solutions to achieve this. Reacting to such challenges, the Dutch government is reformulating its steering philosophy from 'taking care of flood prevention', to 'making sure that the Netherlands should be safe enough against flooding'. The new philosophy involves a transformation of the traditional approach, based on controlling the probability of flooding events, towards a risk based approach, taking into consideration the values protected. The aim is to smoothen the policy process, while downsizing the role of central government, enhancing the role of the citizens and other public and private parties.

The Dutch Ministry of Transport and Water Management recently decided to examine the institutional implications of this new approach for the political and governmental power relations in water management and flood prevention. This paper considers the potential impact on these arrangements of aspects like the perception of vulnerability and risk, public awareness and involvement, culture, in the context of reshuffled responsibilities and power relations.
\end{abstract}

Keywords: water management, flood protection, institutional framework, risk approach, perceptions, public values, the Netherlands.

\section{Introduction: from government to governance?}

In deltas, populations always face flooding risks. For centuries, in the Netherlands, risk management was primarily based on dike enhancement, taming the rivers between ever higher dikes and keeping the sea out. Confronted with 
the high levels of the rivers in the 1990s and resistance of stakeholders against the harmful effects of dike enhancement on cultural and landscape values, policy makers and scientists became involved in critical debates about the required level of security and possible solutions to achieve this. The Dutch defence against flood risks is traditionally institutionalized in hierarchical structures, based on strict rules and pre-established singular indicators and norms. Moreover, the issue of flood protection is also seen in the light of a general reconsideration of Dutch public policy, regarding its responsibilities and role vis-à-vis civil society. The idea of the government as the central and directive actor is questioned.

Facing the 'near flooding' events and acknowledging the consequences of climate change, a rising sea level and subsidence, the Dutch government became conscious of the fact that technical measures, like higher dikes, would no longer be sufficient to prevent flood damages. New, more versatile, ways of dealing with the threats of flooding were to be considered. The responsible Ministry of Transport and Water Management introduced a new perspective: instead of 'taking care of flood prevention' it intends 'to make sure that the Netherlands should be safe enough against flooding'. Its paradigm shifts 'from managing the water and keeping it out', to 'anticipating and adapting to the movement of water'. This new steering philosophy involves a transformation of the traditional approach, based on controlling the probability of flooding events, towards a risk based approach, taking into consideration the values protected and the interested parties. Instead of on technological assignment of hydrological and civil engineering factors, the new approach will draw strongly on organic management or governance.

Yet, upon realizing the political and social complexities of introducing such a new perspective in a deeply embedded facet of Dutch society, the Ministry recently decided to examine the implications of this new approach for the institutions and the political and governmental relations in water management and flood prevention. This paper reports on discussions and interviews with national and regional policymakers and water managers about the approaches and orientations that offer the most likely opportunities for change and transition. The following chapters explore their perspectives on the emerging institutional environment, dilemmas regarding the governance structure and the conditions for implementing the new philosophy. Main issues to be examined are the potential impact of aspects like the perception of vulnerability and risk, public awareness and involvement and culture.

\section{New institutional perspectives: a framework}

The potentials of the new policy arrangements should meet aspects like the perception of vulnerability and risk, public awareness and involvement, culture, reshuffled responsibilities and power relations. A useful conceptual framework is provided by Oliver Williamson [6]. This framework shows that the 'market' is not an abstract mechanism for the coordination of transactions, but that markets are shaped by a set of socially constructed institutions at several levels which influence the behaviour of the actors in these markets. 


\subsection{Williamson's analytical framework}

The framework (see Figure 1) distinguishes four layers of analysis. The top layer, being the most deeply embedded and static, embraces the informal institutions, which influence the formal institutions, like the shape of the polity and the position and approach of judiciary and government bureaucracy. The second level involves the formal rules of the game, most notably with respect to property rights and responsibilities of government agencies.

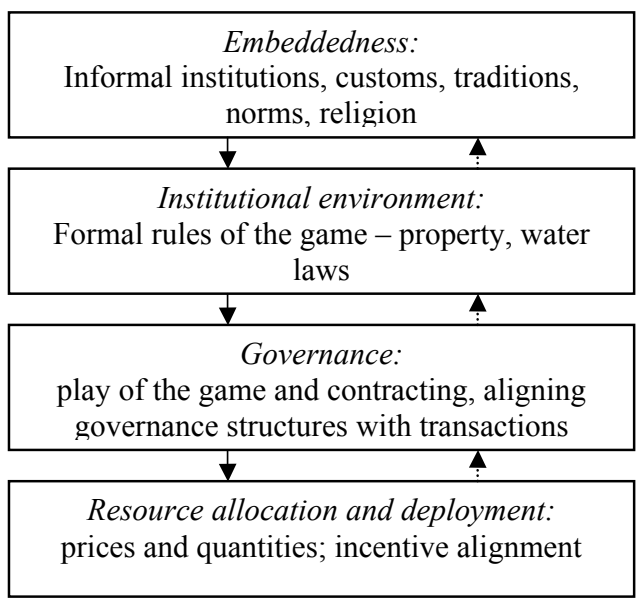

Figure 1: Williamson's layer model [6].

Governance is at the third level of the model, where the actual incentive structures are established that govern the transactions between actors at the lowest level. This may also involve the choice to provide a good, like flooding protection, in a particular public/private relationship. The lowest level, finally, concerns actors' short-term decisions concerning resource allocation, constrained and facilitated by the existing institutions.

The lower layers, entirely operational and most flexible, are influenced, either constrained or facilitated, by the layers above, as indicated by the solid arrows in Figure 1. Nevertheless, developments in the lower layers may affect the higher layers, by means of deliberate attempts of actors, policy makers and politicians to alter the institutions, in response all kinds of developments. This is indicated by the dotted arrows. This, generally, takes time.

\subsection{Williamson's framework and Dutch flood protection}

Interpreting the current developments in Dutch flood management in terms of Williamson's layered model, it can be observed that a number of transitions are taking place, in parallel, at several levels. Interestingly, this implies that we are not only confronted with a unilateral top-down effect of some changing 
(in)formal institutions, like the weakening of equity related principle that each Dutch citizen should have the same level of protection against being flooded. A feedback loop can be discerned, in the sense that the government tries to influence perceptions and beliefs by public campaigns to make citizens aware of flooding risks again, and so draw attention to the responsibility of individuals, instead of the assumption that the government will take care for your safety. Also, there are shifting notions about the balance between central and local decision making, which belong in the legal sphere of the second layer. At the third layer, we do find the increasingly widespread conviction that new arrangements should incentivize civilians and businesses to assume their responsibilities regarding the potential consequences of flooding. At the level of the (trans)actions, new options are being considered, ranging from the idea that civilians should prepare their houses to deal with flood occurrences, including the notion that areas will remain under-protected. In the latter case, refuges and escape paths and the support of emergency services become crucial.

Part of these shifts is driven by the anticipated outcomes of plain cost-benefit analyses, whereas other developments are related with newly acquired physical insights in water related phenomena. Sometimes new visions about effective and efficient government play a role and ideological aspects have an impact. Culture and perceptions of regional populations seem to be important as well. Obviously, this multitude of drivers and effects at the several layers very much complicates the analysis. Finding a direct, clear cut relationship between cause and effect seems way beyond what is feasible. So, our modest objective is to sketch some of the main drivers in this development, to connect these drivers to elements pertaining to the several levels of Williamson and, finally, to identify potential tensions and discrepancies between the roles and interests of actors and the associated institutional structures.

The top layer of the model applies to cultures as a whole and is relatively independent of specific actors. Main elements in this layer are local beliefs about the vulnerability in respect of flooding and historically developed practices of evaluating the options for protection, their effectiveness and costs. Intriguingly, the awareness of much of the Dutch population is quite limited in this respect, as a consequence of the prevailing tradition to organize flood protection in a rather centralized way. A fairly technical, depoliticized, engineering approach has emerged in the $19^{\text {th }}$ century, which has effectively neutralized flooding issues from the local and national political debate. In the 1950s, the disastrous flooding of Zeeland became the main driver for probability based framework that defined the prospective chances of flooding in the several regions of the Netherlands. Keeping the dikes up to a specific strength became the main instrument to achieve these calculated flooding probabilities. Essentially, the whole process of decision-making took place at a large distance from the inhabitants and the main message transmitted to the population was that the state would take care of flood protection.

The second layer, involving the formal institutions, deals with the 'generic' institutions of government; namely, the polity, the judiciary and the bureaucracy. For, example article 21 of the Constitution maintains that the state has to take 
care of the habitability of the country, providing the state with the responsibility to carry out water management and flood protection. It also contains the sectorspecific institutions to deal with flooding risks. The dividing line is not entirely clear, as water management-specific elements are partly taken care of by 'generic' institutions, under auspices of the Ministry of Transport and Water Management, and partly by the regional water boards, responsible for the maintenance of the dijkringen, integral areas surrounded by one dike system with a singular level of protection. It is useful to distinguish 'generic' and 'specific' institutions, because not only the new approach in Dutch water management, but also the EU water and flooding directives, will have an impact on both types of institutions.

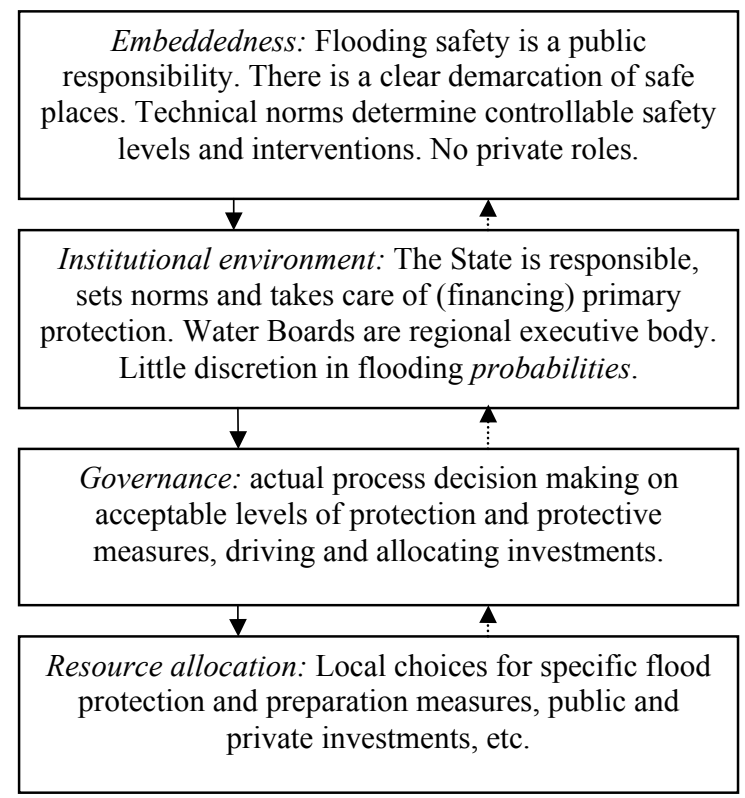

Figure 2: Williamson's layer model, applied to water management.

The third layer is the governance layer, i.e. 'the play of the game'. Officially, this layer has been fairly thin in the past. Indeed, predefined maintenance and construction norms, followed from pre-established flooding probabilities for four types of regions. Local Water Boards monitored their dyke systems and got 'advice' and funding for updating their system. In actual practice, sometimes, budgetary restrictions and local peculiarities in spatial planning turned this 'play' into a situation of negotiation, involving local interests and other public authorities. The bottom layer is where actual resource allocation and investments in flooding protection take place, given the limited room left when the rules of the above layers are applied. Dykes were either or not built and enhanced; provinces and municipalities had adjust their spatial planning objectives. 


\section{Institutional challenges}

The foreseen changes in flood management will have important implications and introduce new tensions within and between institutional layers. In an interactive process of negotiation, discovery and social learning, local and national policy makers, civil servants, experts and water managers are contemplating alternative arrangements to prevent and to react on potential flooding. The assumption is that new policy arrangements should re-balance and re-arrange the degree of protection of particular areas, weighted by population density and economic values at risk.

\subsection{Tension between the governance of prevention and risk management}

In discussing flooding risk, there are both laconic and pessimist professionals, although the decisive voice seems to belong to those who emphasize that the Netherlands has to prepare for flooding and the consequent disruption of society.

In line with the present rules of the game, the responsibility is taken up by the central government. In the context of the new approach, most practitioners relate the redistribution of responsibilities and the effort to enhance 'public' efficiency to a more regionally differentiated water management. The underlying thought is that not all regions are in need of the same level of protection, as should be shown by a cost-benefit analysis. This perspective assumes a far more detailed and economically defined assessment of local risk and protection levels than the current practices. Moreover, many uncertainties have to be dealt with. These insights will have to be translated into drivers to make citizens and firms decide where they prefer to build, given levels of risk and protection provided and to engage local authorities to organize their crisis management approaches.

So far, the new approach has materialized in a public campaign to enhance the public consciousness in respect of flooding risk and a debate about the public role in this respect. Yet, without any floods taking place, it is hard to convince the general public and policy makers to take part in the public debate. Moreover, with the traditional governance of flood prevention placed at the national level, with executive roles delegated to the regional level, flood protection and national safety norms did never receive much political attention, nor were they given much priority in the considerations of local water managers.

\subsubsection{Local impact, governance and flooding disasters}

At the bottom layer of the scheme above, the daily operations of local water managers, the notion of risk instead of probability will link flood prevention to crisis management practices. The new approach towards flooding will be related to the 'generic' management of hazards of major industrial accidents and external safety issues, influenced by the Seveso II decree. A new set of rules has to be developed to engage local and regional authorities in the 'safety chain', which will be replacing the former unilateral approach. This perspective has materialized in a national 'risk map' (www.risicokaart.nl), projecting thirteen potential types of risks with societal impact onto specific areas. Yet, unlike classical disasters, a flooding disaster like in New Orleans has illustrated that the 
operational organization of rescue operations and support should draw on regions adjacent to the flooded area (Wesselink [5]). What is not visible on that map, moreover, is that flooding will often affect more than one vital infrastructure, like electricity, water supply, telecommunications and transport. The interrelatedness of these vital infrastructures makes the operational flooding crisis management even more complex.

Moreover, spatial and hydrological flood characteristics have a major impact on the organization and allocation of crisis management efforts and, thus, differ from traditional 'dry' major accidents. Local conditions have to be taken into account for the limitation of damage, as a primary concern of governance, and for the management of emergency operations, including escape routes and plans, safe places, and the provision of emergency services, energy, hospitals, etc. The modelling of direct damage in detail, allows differentiation on the level of plots of land and roads, instead of areas and infrastructures. Local factors, like the direction and speed of currents, inundation depths, timing, etc. are crucial for local emergence solutions, for protective constructions and for the valuation of risk.

Finally, unlike the current approach, the new policy context assumes decision making on measures for flood prevention and damage control by property developers, construction firms and house owners. In the governance of flood prevention, crisis management, infrastructure maintenance and flooding control should meet.

\subsubsection{Differentiation, valuation and information}

Today, in the governance layer, the public tasks are still defined in terms of dike heights and strengths. Since the land behind the dikes should be safe, these areas and its functions have not been valuated. But in practice, also dike infrastructures have critically weak parts, whereby the probability of bursting, piping and the consequent flooding differs locally. The perspective on the efficiency of current policy practices, and the affordability of water management might change when the risks and values of certain areas get known. The introduction of considerations of efficiency requires the development of new principles and rules for the (e)valuation of local flood risk.

Moreover, in the current perspective on safety, the protection of the land outside the dikes is not a public task. As a consequence, no facts and figures are available about flooding risks and direct and indirect damage in those areas. Counter to intuition, these unprotected areas will relatively soon be accessible again, whereas in inundated areas behind dikes, the water will come fast but takes a lot more time to leave. Consequentially, material and immaterial damage and recovery costs have to be calculated differently. Recovery and revitalization of inundated areas is not in the scope of water management, flood protection and crisis management so far. The question is whether or how this issue should be involved in the debate about the valuation of areas and consequential loss, and related to differentiation of risks between areas.

In the new perspective, it can be expected that the governance layer will become much more important and complex. The codes, rules and decisions are to be negotiated and established between the Ministry of Transport and Water Management, the Water Boards, Provinces, municipalities and other regional 
(safety) authorities. Agents' behaviour in this layer will be strongly influenced by safety indicators and political sensitivities. It will introduce a chain of options in safety management, which also consist of prevention and rescue management by many different organizations with separate tasks and responsibilities.

An important new element of this layer will be the development of public/private financing mechanisms for flood prevention and preparedness, in the light of economic and social differentiation between functions and areas, and the degree to which safety or risk on flooding is considered optimal.

\subsubsection{Flood protection governance in an institutionally fragmented environment}

In the formal institutional layer, in the infrastructures sectors, governments are pulling away from ownership and management. The central government tends towards a role of coordinating networks and markets and oversight. Thus, the governance of flood protection gets fragmented in various ways by the shift towards the risk approach. The responsibility for safety and risk management will be reallocated among a larger set of actors, many with competing goals and interests and diverging and often conflicting public values like security and privacy. Two important questions arise: could this fragmented infrastructure be still reliable? And could the parties involved kept responsible? The exact relationship between institutional restructuring and the reliability of infrastructures is largely neglected, still (WRR [3]). In respect of water management and flood protection, at least three issues seem to count.

Firstly, borders of institutions, authority and power are scaled differently than the dike system. Next to the organizational complexity of networked flood protection, this may implicate that fall-back options are in the domain of other parties. This may have consequences for the public safety. Also political and economic interests may become increasingly important in the definition of 'public values'. Without involvement of a final decisive authority, agreements and trade offs will have to be negotiated, between flooding control, prevention related measures and other objectives. The role of private parties in flood protection and dike maintenance, like in other competitive sectors, will give rise to divergent and conflicting public and private interests. Yet, in the end, the central government will retain, formally or informally, responsibility for the consequences of floods and flood protection.

Secondly, the parallel transition in the water policy and the steering philosophy causes a discrepancy between a fuzzy 'safety' problem at the operational and governance level and strictly defined, extensive public institutional responsibility. Politicians try to alter the rules of the game, by making authorities and citizens aware of this shift. Indeed, the new Dutch Water Act (2008 forthcoming) reallocates responsibilities towards individual citizens, while the institutional environment should be adjusted to encompass a life with water, instead of a battle against water. New rules will have to incorporate new definitions, interpretations and arrangements for insurances, liabilities and the responsibility of public and private actors.

Finally, it is obvious that public policy is now 'making sure that the Netherlands should be safe enough against flooding'. Whereas the risk approach 
considerably affects the citizens' perspective on flooding, it does not really alter the viewpoint of politicians; they still prefer to send the message that it is safe all over the place. In this context, it will be a major challenge to credibly and effectively deal with uncertainties about (modelling) risks and (economization of) public values.

\subsubsection{Tension between the individual and the public}

As regards the informal, culturally defined layer, public awareness is drawn to the risk approach by a campaign to enhance the citizen's consciousness in respect of flooding. In the Dutch debate, arguments about the individual interest in flood prevention and the public responsibility are often intermingled. Although the protected values may look the same: public values have different weight in particular, regional, local and individual contexts and should be operationalised in different ways. The several private values, in turn, can not be aggregated upwards consistently. Water gives individuals a sense of freedom and joy. Everyone is attracted by beautiful accessible waterfronts. But their safety is a collective concern.

This raises the question of the authority's expectations regarding citizen behaviour. Citizens' perceptions of flooding risks do become more relevant in the new steering context; not as a perception to be 'adjusted' in the short term, but as a powerful social force to take into account. Operational management will have to face perceptive individuals. Firstly, whereas floods and possible emergency operations can be foreseen in advance, it is known that local rescue workers will have their own priorities and families, competing with their professional ethos. Secondly, in the different public risk and security approaches, citizens are either involved, excluded, informed, instructed, claimed, or held responsible in different ways and for all kinds of tasks in water management and flooding prevention. Often, however, the individuals working and living in risky areas are unaware of what the different public authorities are precisely expecting from them and what they can expect, in return. After having read in the yesterday's newspaper that new measures will provide safety to all, the flyer in the mail today urges to be prepared for a flooding catastrophe, by regularly refreshing iron rations and batteries and by keeping an inflatable dinghy in the attic. This is not only weak communication policy; it emerges from authorities diverging expectations and underlying assumptions in respect of individual's perceptions.

\section{Concluding remarks}

The new steering philosophy implies that both the technological problem definition and the organizational approach become more complex. In the governance of flood prevention, crisis management, infrastructure maintenance and flooding control should meet. However, the most important tensions are expected at the interfaces between the layers of the analytical framework. All respondents at all layers cope with a fuzzy problem definition, interpreted from their own perspectives and context. 
On the one hand these changes involve the rules of the game. Governmental responsibility for probability of flooding events is currently institutionalized in strictly separated centralized organizations, relatively immune from local and national politics. But, at the formal level, the Environmental Licensing Bill, the Spatial Planning Act and the Water Act are under review. As authorities and power relations will be shifting, new measures to protect against flooding will develop in a 'newly' or differently institutionalized policy domain.

On the other hand, the play of the game will be changing as well. Traditionally, public water managers are not used to operate close to others. The Water Boards and the government have specialized in specific tasks in a vertical and horizontal mode of organization, while their focus regarding communication and decision making is on the above-regional level. The recent initiatives have made these organizations aware of the fact that will have to cooperate and share responsibilities with provinces and municipalities, which are much more focused on citizens. Yet, there are large differences in cultures, structures, traditions, habits and calculative strategic behaviour.

The parallel transitions cause a discrepancy between a 'safety' problem at the operational and governance level and strictly defined, extensive public institutional responsibility. The governance layer will become much more important and complex, and the operational layer much more dispersed. Due to different dynamics in both layers a smooth process will be 'a bonus for fast decision makers'. The implementation of the new principles on regional levels may (e.g. Brink and Meijerink [1]) encounter strong resistance in getting acceptation. Indeed, traditionally, the acceptation of water management in the Netherlands was based on notions like: explainable, affordable, enforceable, feasible, widely supported and sustainable. In the future, the meaning of these notions will have to change, as flooding experiences, or the lack thereof, may be socially articulated, politicized and will be affecting the above institutional layers, in a social learning process.

The risk approach will not really alter the viewpoint of politicians focussing on citizens' perceptions. They should be interested in the analyses of perceptions but as dynamic instrument, to follow the development of the complexity of the institutional context. An image of perceptions, interests, power and dependencies can help institutional actors to operationalise and guarantee public values in a meaningful and accepted way. Their major challenge to credibly and effectively is to deal with uncertainties about (modelling) risks and (economization of) public values. A stated above, new policy conditions and dilemma's require reconsideration of safeguarding public values. Lessons from other critical infrastructure sectors can be learned (Bruijn and Dicke [2]), with a natural focus on safety and reliability, like transport, electricity and ICT infrastructures where totally different policy arrangements are in use. A glance at the general and specific problems in other sectors might as well be helpful for approaching the interconnectivity issues and an overall risk approach, with respect to public values and responsibilities. The shift to a risk approach might have implications for decisive arguments like acceptable risk, indicators, continuity and reliability. Concepts like marginal reliability and reliability of precluded events (Schulman 
et al [4]) can renew the debate about shared responsibilities. A changing perspective might direct to differentiate policy on flooding risks and reliability of dike infrastructures.

This paper is developed as part of the research 'Perceptions and risk communication in dealing with flood risk' (P3062-2) and with funding from the 'Living with water' program.

\section{References}

[1] Brink, M. van den \& Meijerink, S., Implementing policy innovations resource dependence, struggle for discursive hegemony and institutional inertia in the Dutch river policy domain, Working paper series 2006/2, Radboud University Nijmegen, 2006

[2] Bruijn, H. de \& Dicke, W, Strategies for safeguarding public values in liberalized utility sectors, Public administration, 84/3, pp. 717-735, 2006.

[3] WRR (scientific council for government policy), Water management and water safety, web publication no.39 (in Dutch), 2007

[4] Schulman, P., Roe, E., Van Eeten, M. \& De Bruijne, M., Reliability in real time: reliability theory and the management of complex systems, Administrative science quarterly, under review, 2002

[5] Wesselink, A.J., Bijker, W.E., De Vriend, H.J., Krol, M.S., Dutch dealings with the Delta, Nature and Culture, 2/2, pp. 188-209, 2007.

[6] Williamson, O.E., Transaction Costs economics: How it Works, where is it Headed, The Economist, 146/1, pp. 23-58, 1998. 